\title{
RELICS OF NUCLEAR ACTIVITY: DO ALL GALAXIES HAVE MASSIVE BLACK HOLES?
}

\author{
ROELAND P. VAN DER MAREL \\ STScI, 3700 San Martin Drive, Baltimore, MD 21218, USA
}

\begin{abstract}
The distribution of black hole (BH) masses $M_{\bullet}$ in galaxies is constrained by photometric and kinematic studies of individual galaxies, and by the properties of the quasar population. I review our understanding of these topics, present new results of adiabatic $\mathrm{BH}$ growth models for $H S T$ photometry of elliptical galaxies with brightness profiles of the 'core' type, and discuss the implications of ground-based stellar kinematical data. It is not yet possible to uniquely determine the $\mathrm{BH}$ mass distribution, but the available evidence is not inconsistent with a picture in which: (i) a majority of galaxies has BHs; (ii) there is a correlation (with large scatter) between $M_{\bullet}$ and spheroid luminosity $L_{\mathrm{sph}}$ of the form $M_{\bullet} \approx 10^{-2} L_{\mathrm{sph}}$ (solar B-band units); and (iii) the BHs formed in a quasar phase through mass accretion with efficiency $\epsilon \approx 0.05$.
\end{abstract}

\section{Introduction}

Considerable evidence suggests that the energetic processes in active galaxies and quasars are due to the accretion of matter onto massive BHs. Lynden-Bell (1969) already suggested that BHs may also be present in quiescent galaxies, such as the Milky Way, M31 and M32. This spurred efforts to find BHs in nearby galaxies through kinematical studies, which have since increased steadily in sophistication, both observationally and theoretically. There are now convincing $\mathrm{BH}$ detections for at least a dozen galaxies, and new detections are reported at an ever increasing rate. The techniques for detecting $\mathrm{BHs}$ in individual galaxies have been reviewed by, e.g., Kormendy \& Richstone (1995, hereafter KR95), Ford et al. (1998) and Richstone (1998). Here I address the more general question: do all galaxies have BHs? 


\section{Quasar counts and evolution}

Integration of quasar number counts yields the comoving energy density in quasar light. Assuming that this energy is produced by accretion onto massive black holes, one obtains the total mass per cubic Mpc that is collected in black holes (Soltan 1982). Division by the observed luminosity density of galaxies (Loveday et al. 1992) yields an estimate of the average black hole mass per unit luminosity: $\left\langle M_{\bullet}\right\rangle /\langle L\rangle=2.0 \times 10^{-3}(0.1 / \epsilon)$, where $\epsilon$ is the accretion efficiency (Chokshi \& Turner 1992). [Throughout this paper, $H_{0}=80 \mathrm{~km} \mathrm{~s}^{-1} \mathrm{Mpc}^{-1}$, mass-to-light ratios are in solar units, and luminosities are in the B-band.]

To address the $\mathrm{BH}$ mass distribution, one must model not only the total energy budget of the quasar population, but also its evolution. Tremaine (1996; also Faber et al. 1997, hereafter F97) presented a simple argument based on the typical quasar lifetime to show that a model in which every spheroid (bulge or elliptical) has a $\mathrm{BH}$ is consistent with the inferred $\left\langle M_{\bullet}\right\rangle /\langle L\rangle$. Haehnelt \& Rees (1993, hereafter HR93) presented a more detailed model (in which $\mathrm{BH}$ formation is linked to hierarchical structure formation) to fit the distribution of quasars as function of magnitude and redshift. Their predicted $\mathrm{BH}$ mass distribution at the current time (their Fig. 8 ) is consistent with a fraction $f \approx 0.3$ of all galaxies having a $\mathrm{BH}$.

The uncertainties in these estimates are considerable. The only conclusion that can be drawn with some confidence is that a fraction $f=0.1-1$ of all galaxies is likely to contain BHs with $M_{\bullet} / L=10^{-2}-10^{-3}$. The product of these quantities, $\left\langle M_{\bullet}\right\rangle /\langle L\rangle$, is better constrained than either quantity independently, but is still rather uncertain (if only due to the unknown $\epsilon$ ).

\section{BH detections}

HR93 predict a direct connection between $M_{\bullet}$ and the galaxy formation redshift $z_{\text {form }}$ (galaxies that form later have smaller BHs), but not between $M_{\bullet}$ and galaxy luminosity. Spirals form later than ellipticals, and are therefore predicted to have smaller BHs. Observations appear to confirm this; e.g., the (active) galaxies M87 and NGC 1068 have similar luminosities, but $M_{\bullet}$ is $10^{2.5}$ times larger in M87 (cf. Figure 1a below). Observations do not rule out a correlation between $M_{\bullet}$ and the spheroid luminosity of the host (KR95). This may indicate that $M_{\bullet}$ and $L_{\text {sph }}$ depend similarly on a common underlying parameter (e.g., $z_{\text {form }}$, as in the models of HR93), or alternatively, that there is a physical link between BHs and spheroids.

Figure 1a shows $M$. versus $L_{\text {sph }}$ for all currently available BH mass determinations inferred from: $(x)$ radio observations of water masers; (o) ionized gas kinematics of nuclear disks; $(*)$ time variability of broad doublepeaked Balmer lines; and $(\bullet)$ stellar kinematical studies that included anisotropic modeling (studies with only isotropic models are discussed in $\S 6$ ). 


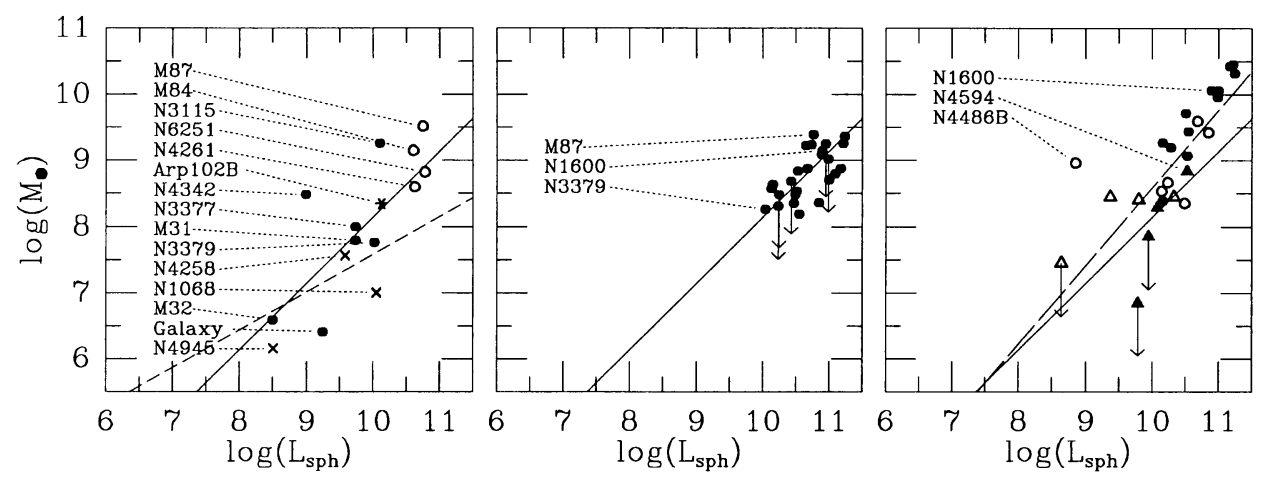

Figure 1. Measurements of black hole mass $M_{\bullet}$ versus spheroid luminosity $L_{\mathrm{sph}}$. Arrows indicate upper limits. (a; left) 'Secure' BH detections obtained with various techniques, as indicated by different symbols and discussed in $\S 3$. References are included in the bibliography; where necessary, spheroid/total luminosity ratios were estimated from the Hubble type and the relation in Simien \& de Vaucouleurs (1986). (b; middle) $M_{\bullet}$ values for all core galaxies in F97 with $M_{\mathrm{B}}<-20$, inferred from models of adiabatic $\mathrm{BH}$ growth for $H S T$ photometry ( $\$ 4$; van der Marel 1998, in preparation). M87 and NGC 3379 are the two core galaxies for which secure kinematic determinations are also available (cf. panel a). NGC 1600 is discussed in $\S 5$. (c; right) $M_{\bullet}$ determinations from axisymmetric $f\left(E, L_{\mathbf{z}}\right)$ models for ground-based stellar kinematical observations of elliptical galaxies (Mg98). Circles: 'core' galaxies; triangles: 'power-law' galaxies; open symbols: galaxies in Virgo. Galaxies already included in the left panel are omitted. For NGC 4486B and 4594 the presence of a $\mathrm{BH}$ has been suggested previously on the basis of isotropic models. The $M_{\bullet}$ values in panel a are typically believed to have $\left|\Delta \log M_{\bullet}\right| \leq 0.3$. The accuracy of the $M_{\bullet}$. measurements in panels $\mathrm{b}$ and $\mathrm{c}$ is dominated by systematic uncertainties in the model assumptions, as discussed in $\S 4,5$ and 6 . In particular, mild radial velocity anisotropy would lower the values in panel c. The solid line in each panel is the 'reference model' discussed in the $\S 3$. It has $M_{\bullet}=1.4 \times 10^{-2} L_{\mathrm{sph}}$, and fits the constraints from quasar number counts for an assumed accretion efficiency $\epsilon=0.05$. The long-dashed line in the rightmost panel is an alternative model. It has $M_{\bullet}=6.0 \times 10^{-3} M_{\mathrm{sph}}$ (with $M_{\mathrm{sph}} \propto L_{\mathrm{sph}}{ }^{1.18}$, cf. Mg98), and fits the constraints from quasar number counts for $\epsilon=0.022$. The dashed line in the left panel indicates those $M_{\bullet}$ for which $r_{\bullet}=0.1^{\prime \prime}$ at $D=10 \mathrm{Mpc}$.

There is indeed a correlation, but the scatter is large $(\sim 2$ dex at fixed $L_{\text {sph }}$ ) and selection bias may be important. The dashed line shows the $M_{\bullet}$ for which the $\mathrm{BH}$ sphere of influence, $r_{\bullet} \simeq G M_{\bullet} / \sigma^{2}$, extends $0.1^{\prime \prime}$ at a distance $D=10 \mathrm{Mpc}$ ( $\sigma$ is determined by $L_{\text {sph }}$ through the Faber-Jackson relation). BHs below this line can be detected only in galaxies closer than $10 \mathrm{Mpc}$, and in galaxies in which kinematical tracers can be observed at resolutions $<0.1^{\prime \prime}$ (e.g., water masers).

The solid line shows the predictions of one possible model that is consistent with the $\left\langle M_{\bullet}\right\rangle /\langle L\rangle$ from quasar number counts. This 'reference model' assumes that every spheroid has a BH with $M_{\bullet} \propto L_{\mathrm{sph}}$. Approximately $30 \%$ of the light from galaxies is due to spheroids (Schechter \& Dressler 1987). So for an assumed accretion efficiency $\epsilon=0.05$ this yields $M_{\bullet}=1.4 \times 10^{-2} L_{\mathrm{sph}}$, which reproduces the trend in the data. 


\section{Surface-brightness profiles}

$H S T$ observations of early-type galaxies reveal central surface brightness cusps that fall in two categories (F97), 'power-laws' (showing no clear break) and 'cores' (showing a clear break). Cusps can be explained as a consequence of the influence of a $\mathrm{BH}$ on surrounding stars (Young 1980, hereafter Y80). Properties of cusps around BHs depend on $M_{\bullet}$, initial conditions (Quinlan et al. 1995, hereafter Q95) and two-body relaxation (Bahcall \& Wolf 1976). Cusps may also be due to processes unrelated to BHs (KR95), and they can also be destroyed (Quinlan \& Hernquist 1997). Observed cusps therefore do not uniquely constrain the $\mathrm{BH}$ masses in galaxies.

Nonetheless, simple models of adiabatic BH growth for observed photometry of M87 (Young et al. 1978; Lauer et al. 1992; Crane et al. 1993) and several other galaxies imply $\mathrm{BH}$ masses that agree well with kinematic determinations. I have therefore started a study of adiabatic BH growth models for a large sample of galaxies with published HST photometry. These models may be particularly relevant for core galaxies, for which the observed break in the brightness profile may be associated with an originally homogeneous core. I have used the software of Q95 to fit the photometric models of Y80 to all core galaxies in the sample of F97 with $M_{\mathrm{B}}<-20$ (van der Marel 1998 , in preparation). The models fit well in the central few arcsec (RMS residual $\left.\sim 0.05 \mathrm{mag} / \operatorname{arcsec}^{2}\right)$, and the photometrically inferred $M_{\bullet}$ appear meaningful: the kinematically determined $M_{\bullet}$ for M87 and NGC 3379 (see Figure 1a) are reproduced to within 0.12 and 0.50 dex, respectively.

Figure $1 \mathrm{~b}$ shows the results for the whole sample. The $M_{\bullet}$ are remarkably consistent with the kinematical detections in Figure 1a, and show a similar trend with $L_{\mathrm{sph}}$. So despite their simplicity, it may well be that the Y80 models capture the essence of surface brightness cusps in core galaxies. The prevalence of these cusps would then imply that most or all core galaxies have BHs, with $M_{\bullet} \propto L_{\text {sph }}$ as suggested by Figure $1 \mathrm{~b}$.

\section{Stellar kinematics and velocity dispersion anisotropy}

Stellar motions often provide the only kinematical tool to study BH masses in quiescent galaxies, but the well-known degeneracy between $M_{\bullet}$ and velocity dispersion anisotropy (Binney \& Mamon 1982) is still a major complication. This degeneracy can be resolved when high resolution HST data are available (e.g., van der Marel et al. 1997; Gebhardt et al. 1998), but such data are not yet available for many galaxies. Lower resolution groundbased data are plentiful, but more ambiguous to interpret. I use the case of NGC 1600, an E3 core galaxy with no significant rotation, to illustrate this.

Ground-based kinematical data with $\sim 2^{\prime \prime}$ resolution (Jedrzejewski \& Schechter 1989) show a mildly peaked velocity dispersion profile, and HST 

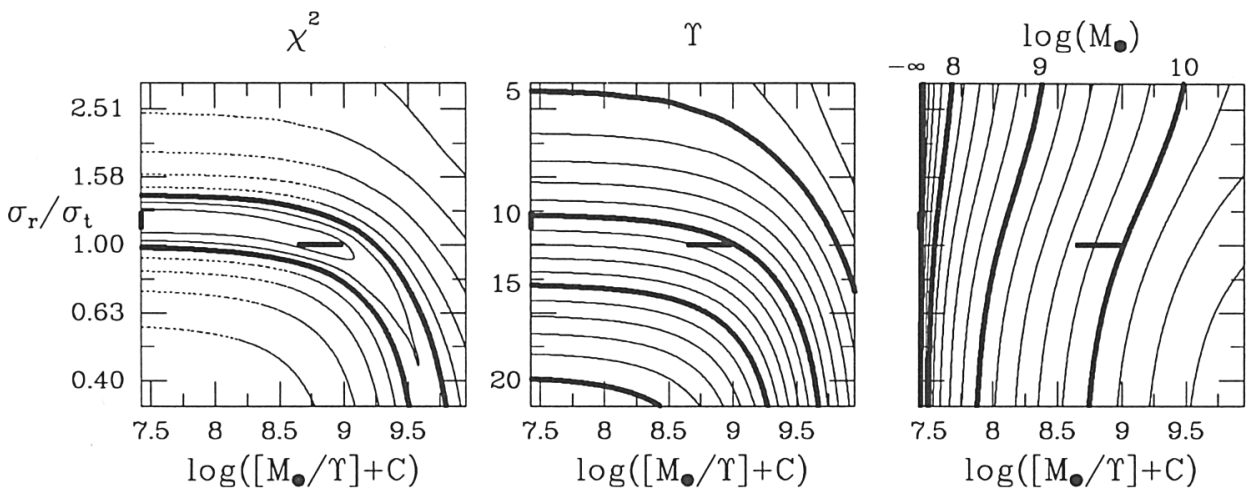

Figure 2. Predictions of spherical dynamical models for NGC 1600 that reproduce HST and ground-based photometry (F97; Peletier et al. 1990). The abscissa in each panel is $\log \left(\left[M_{\bullet} / \Upsilon\right]+C\right)$, where $\log C \equiv 7.425$; i.e., approximately logarithmic in $\left(M_{\bullet} / \Upsilon\right)$ for $\left(M_{\bullet} / \Upsilon\right) \gg C$, and $M_{\bullet}=0$ on the left boundary. The ordinate is $\sigma_{\mathrm{r}} / \sigma_{\mathrm{t}}$, increasing logarithmically from $1 / 3$ to 3 . For each $\left(M_{\bullet} / \Upsilon ; \sigma_{\mathrm{r}} / \sigma_{\mathrm{t}}\right)$, the stellar mass-to-light ratio $\Upsilon$ was chosen to minimize the $\chi^{2}$ of the fit to the kinematical data of Jedrzejewski \& Schechter (1989). (a; left) Contours of $\chi^{2}$. The first two contours correspond to the $1 \sigma$ and $2 \sigma$ level, as calculated from the statistic $\Delta \chi^{2}$. The heavy contours show the $3 \sigma$ region. (b; middle) Contours of $\Upsilon$, increasing linearly. (c; right) Contours of $M_{\bullet}$, increasing logarithmically. Heavy curves in panels $b$ and $c$ indicate contours for which the value of $\Upsilon$ or $\log M_{\bullet}$ is indicated. Contours of $\Upsilon$ and $\chi^{2}$ are approximately parallel, so $\Upsilon$ is well determined independent of the anisotropy, $\log \Upsilon=1.02$. By contrast, contours of $M_{0}$ and $\chi^{2}$ are approximately perpendicular, so there is a strong degeneracy between $M_{\text {. }}$ and anisotropy. In all panels, a heavy horizontal bar indicates those isotropic models with a $\mathrm{BH}$ that are acceptable at the $1 \sigma$ level, and a heavy vertical bar indicates those anisotropic models without a $\mathrm{BH}$ that are acceptable at the $1 \sigma$ level.

photometry shows a shallow (F97), marginally significant (Byun et al. 1996; Gebhardt et al. 1996), surface brightness cusp. I construct spherical dynamical models (adequate for the present purpose) following the approach of van der Marel (1994, hereafter vdM94). I solve the Jeans equation for a given velocity anisotropy profile profile, $\sigma_{\mathrm{r}} / \sigma_{\mathrm{t}}(r)$ (where $2 \sigma_{\mathrm{t}}{ }^{2} \equiv \sigma_{\theta}^{2}+\sigma_{\phi}^{2}$ ), project and convolve the results, and compare with the data in a $\chi^{2}$ sense. The normalization of the dispersion profile is determined by the stellar mass-to-light ratio $\Upsilon$, and its shape is determined by $\sigma_{\mathrm{r}} / \sigma_{\mathrm{t}}$ and $M_{\bullet} / \Upsilon$.

Figure 2 shows the fitted $\chi^{2}, M_{\bullet}$ and $\Upsilon$ in the $\left(M_{\bullet} / \Upsilon ; \sigma_{\mathrm{r}} / \sigma_{\mathrm{t}}\right)$ parameter space, for models with constant $\sigma_{\mathrm{r}} / \sigma_{\mathrm{t}}$. The valley in the $\chi^{2}$ contours shows that a one-parameter family of $\left(M_{\bullet} / \Upsilon ; \sigma_{\mathrm{r}} / \sigma_{\mathrm{t}}\right)$ combinations fits the data. An isotropic model requires a very massive $\mathrm{BH}, \log M_{\bullet}=9.85$, but only modest anisotropy, $\sigma_{\mathrm{r}} / \sigma_{\mathrm{t}}=1.18$, is required to fit the data without a $\mathrm{BH}$. Models that are radially anisotropic at all radii may not correspond to a positive phase-space distribution function (DF) (vdM94), or may not be stable (Stiavelli et al. 1993), so I also constructed models in which the core is isotropic, and in which there is a smooth transition at the break radius of the surface brightness profile $\left(r_{\mathrm{b}}=3.12^{\prime \prime}\right)$ to a value $\left(\sigma_{\mathrm{r}} / \sigma_{\mathrm{t}}\right)_{\text {main }}$ characteristic of the main body. Models with $\log M_{\bullet}<10.01$ are still all acceptable at 
the $1 \sigma$ level. The best fit with no BH has $\left(\sigma_{\mathrm{r}} / \sigma_{\mathrm{t}}\right)_{\text {main }}=1.45$, and the best fit with $\log M_{\bullet}=9.15$, as suggested by adiabatic BH growth (Figure $1 \mathrm{~b}$ ), has $\left(\sigma_{\mathrm{r}} / \sigma_{\mathrm{t}}\right)_{\text {main }}=1.30$. So in the absence of independent constraints on the velocity dispersion anisotropy, the data do not significantly constrain $M_{\bullet}$.

Our understanding of the velocity anisotropy in galaxies is only rudimentary. Ellipticals and bulges with power-law brightness profiles have low to intermediate luminosity (F97), and are flattened by rotation. The tensor virial theorem indicates that they may be isotropic. Any anisotropic model with the same average $\left(\sigma_{\mathrm{R}}^{2}+\sigma_{\phi}^{2}\right) / \sigma_{\mathrm{z}}^{2}$ is also viable, but both M32 (van der Marel et al. 1998) and the Galactic Bulge (Evans \& de Zeeuw 1994) have indeed been shown to be nearly isotropic. By contrast, core galaxies like NGC 1600 have intermediate to high luminosity, and little rotation (F97). Detailed studies of three individual galaxies (Merritt \& Oh 1997; Rix et al. 1997; Gerhard et al. 1998) are consistent with mild radial anisotropy, $\sigma_{\mathrm{r}} / \sigma_{\mathrm{t}}=1.2-1.4$, with a possible transition to isotropy at small radii. Such a velocity distribution can be produced by dissipationless collapse (van Albada 1982). Mild radial anisotropy is also consistent with studies of the ratio of major to minor axis kinematics (van der Marel 1991) and lineof-sight velocity profile shapes (Bender et al. 1994) in a larger sample of core galaxies, and is also seen in the Galactic halo in the solar neighbor$\operatorname{hood}\left(\sigma_{\mathrm{r}} / \sigma_{\mathrm{t}}=1.5 \pm 0.2\right.$; Beers \& Sommer-Larsen 1995). So it appears that power-law galaxies may be approximately isotropic and that core galaxy may be mildly radially anisotropic, but neither result is firmly established.

\section{Isotropic models for stellar kinematical data}

Magorrian et al. (1998, hereafter Mg98) studied 36 (mostly) elliptical galaxies for which $H S T$ photometry and ground-based stellar kinematics have been published. Each galaxy was modeled with the Jeans equations, assuming an $f\left(E, L_{\mathrm{z}}\right)$ DF (the axisymmetric generalization of a spherical isotropic model). Figure $1 \mathrm{c}$ shows the $\mathrm{BH}$ masses that best fit the observed kinematics. BHs are required in nearly all galaxies, with $M_{\bullet} \approx 6 \times 10^{-3} M_{\mathrm{sph}}$ (long-dashed line), consistent with quasar number counts if the accretion efficiency $\epsilon=0.022$. This is the first dynamical study that addresses a large sample in a homogeneous way while including $H S T$ photometry. It establishes the important fact that the presence of a $\mathrm{BH}$ in every spheroid is consistent with kinematical data, and that the required $\mathrm{BH}$ masses are consistent with quasar counts for a reasonable value of $\epsilon$.

Nonetheless, the Mg98 results are not unique. Of the 29 galaxies that require a $\mathrm{BH}$ under the $f\left(E, L_{\mathrm{z}}\right)$ hypothesis, 19 are core galaxies with similar data as for NGC $1600 . \mathrm{Mg} 98$ find $\log M_{\bullet}=10.07$ for NGC 1600 , but the results in $\S 5$ showed that all $M_{\bullet}$ smaller than this are equally acceptable. 
So the Mg98 models may have overestimated the masses and/or prevalence of BHs. This would not violate the constraints from quasar counts: if one assumes a higher $\epsilon=0.1$, one may decrease all $M_{\bullet}$ by a factor 4.5 , or remove the $\mathrm{BHs}$ in $78 \%$ of the galaxies.

Two core galaxies in the sample have $M_{\bullet}$ determinations from independent sources. Neither is well fit by an $f\left(E, L_{z}\right)$ model. For M87, Mg98 infer the same $M_{\bullet}$ as inferred from $H S T$ gas kinematics, but only if the data outside $5^{\prime \prime}$ are ignored. For NGC $3379, \mathrm{Mg} 98$ infer an $M_{\bullet}$ that exceeds the more accurate determination of Gebhardt et al. (1998) by a factor 7 . Independent of whether one views these comparisons as reasonable or poor agreement, it leaves open the question whether $f\left(E, L_{\mathrm{z}}\right)$ models return the correct result for galaxies that may not have a (significant) BH.

One may wonder whether the correlation between $M_{\bullet}$ and $L_{\text {sph }}$ inferred by $\mathrm{Mg} 98$ can be explained if the $M_{\bullet}$ values were partly spurious. This is in fact the case. For galaxies that are radially anisotropic, isotropic models will fit the observed dispersion gradients by invoking BHs for which $r_{\bullet} \equiv G M_{\bullet} / \sigma^{2}$ is similar to the observational resolution. This predicts a correlation of $M_{\bullet}$ with distance of the form $r_{\bullet} \approx 2^{\prime \prime}$, which is not inconsistent with the $\mathrm{Mg} 98$ results. The more distant galaxies in the sample are the most luminous. So this predicts not only the correlation of $M_{\bullet}$ with $L_{\mathrm{sph}}$, but also that this correlation should be weaker for the galaxies in Virgo (which are all at the same distance), as seen in Figure 1c.

Actual measurements of the velocity anisotropy are required to establish whether or not the $M_{\bullet}$ inferred by $\mathrm{Mg} 98$ are correct. Either way, the $M_{\bullet}$ in Figure 1c are 4.5 times higher than those in Figure 1b, averaged over the 14 galaxies common to both samples. So either the photometric measurements are too low (not impossible, cf. the uncertainties discussed in $\S 4$ ), or the $\mathrm{Mg} 98$ results are too high (which would require mild radial anisotropy that is not inconsistent with our understanding of core galaxies, cf. §5).

\section{Conclusions}

Our understanding of the $\mathrm{BH}$ mass distribution is still incomplete, partly due to a lack of complete representative samples that cover quiescent and active galaxies of all Hubble types, and partly due to persistent uncertainties in the correct interpretation of photometric and kinematic data. However, it is clear that we are finding $\mathrm{BHs}$ in the correct mass range to explain quasar fueling and evolution, to within the uncertainties.

I thank Gerry Quinlan for kindly allowing me to use his adiabatic BH growth software. This work benefited from discussions with Eric Emsellem, Tod Lauer, John Magorrian, Scott Tremaine and Tim de Zeeuw. It was supported by STScI grant HF-1065.01-94A and an STScI Fellowship. STScI is operated by AURA Inc., under NASA contract NAS5-26555. 


\section{References}

Bahcall J.N., Wolf R.A., 1976, ApJ, 209, 214

Beers T.C., Sommer-Larsen J., 1995, ApJS, 96, 175

Bender R., Saglia R.P., Gerhard O.E., 1994, MNRAS, 269, 785

Binney J., Mamon G.A., 1982, MNRAS, 200, 361

Bower G.A., et al., 1997, ApJL, in press; astro-ph/9710264 [M84]

Byun Y.-I., et al., 1996, AJ, 111, 1889

Chokshi A., Turner E.L., 1992, MNRAS, 259, 421

Crane P., et al., 1993, AJ, 106, 1371

Evans W., de Zeeuw P.T., 1994, MNRAS, 271, 202

Faber S.M., et al., 1997, AJ, in press; astro-ph/9610055 [F97]

Ferrarese L., Ford H.C., Jaffe W., 1996, ApJ, 470, 444 [NGC 4261]

Ferrarese L., Ford H.C., Jaffe W., 1998, ApJ, submitted [NGC 6251]

Ford H.C., et al., 1998, in Proc. IAU Symp. 184 (Kluwer), in press; astro-ph/9711299

Gebhardt K., et al., 1996, AJ, 112, 105

Gebhardt K., et al., 1998, AJ, submitted

Genzel R., Eckart A., Ott T., Eisenhauer F., 1997, MNRAS, 291, 219 [Galaxy]

Gerhard O.E., et al., 1998, MNRAS, submitted; astro-ph/9710129

Greenhill L.J., Gwinn C.R., Antonucci R., Barvainis R., 1996, ApJ, 472, L21 [NGC 1068]

Greenhill L.J., Moran J.M., Herrnstein J.R., 1997, ApJ, 481, L23 [NGC 4945]

Haehnelt M.G., Rees M.J., 1993, MNRAS, 263, 168 [HR93]

Jedrzejewski R., Schechter P.L., 1989, AJ, 98, 147

Kormendy J., Richstone D., 1995, ARA\&A, 33, 581 [KR95]

Kormendy J., et al., 1996, ApJ, 459, L57 [NGC 3115]

Kormendy J., et al., 1996, ApJ, 473, L91 [NGC 4594]

Kormendy J., et al., 1997, ApJ, 482, L139 [NGC 4486B]

Lauer T.R., et al., 1992, AJ, 103, 703

Loveday J., Peterson B.A., Efstathiou G., Maddox S.J., 1992, ApJ, 390, 338

Lynden-Bell D., 1969, Nature, 223, 690

Macchetto F., Marconi A., Axon D.J., Capetti A., Sparks W., 1997, ApJ, 489, 579 [M87]

Magorrian J., et al., 1998, AJ, in press; astro-ph/9708072 [Mg98]

Merritt D., Oh S.P., 1997, AJ, 113, 1279

Miyoshi M., et al., 1995, Nature, 373, 127 [NGC 4258]

Newman J.A., et al., 1997, ApJ, 485, 570 [Arp 102B]

Pei Y.C., et al., 1998, ApJ, submitted [M31]

Peletier R.F., Davies R.L., Lindsey E, Illingworth G.D., Cawson M., 1990, AJ, 100, 1091

Quinlan G.D., Hernquist L., Sigurdsson S., 1995, ApJ, 440, 554 [Q95]

Quinlan G.D., Hernquist L., 1997, New Astronomy, submitted; astro-ph/9706298

Richstone D., 1996, BAAS, 189, 111.02 [NGC 3377]

Richstone D., 1998, in Proc. IAU Symp. 184 (Kluwer), in press

Rix H.-W., de Zeeuw P.T., Cretton N., van der Marel R.P., Carollo, 1997, ApJ, 488, 702

Schechter P.L., Dressler A., 1987, AJ, 94, 563

Simien F., de Vaucouleurs G., 1986, ApJ, 302, 564

Sołtan A., 1982, MNRAS, 200, 115

Stiavelli M., Møller P., Zeilinger W.W., 1993, A\&A, 277, 421

Tremaine S., 1996, in 'Unsolved Problems in Astrophysics', (Princeton Univ. Press), 137 van Albada T.S., 1982, MNRAS, 201, 939

van den Bosch F., 1997, PhD thesis (Leiden University) [NGC 4342]

van der Marel R.P., 1991, MNRAS, 253, 710

van der Marel R.P., 1994, MNRAS, 270, 271 [vdM94]

van der Marel R.P., de Zeeuw P.T., Rix H.W., Quinlan G.D., 1997, Nature, 385, 610

van der Marel R.P., et al., 1998, ApJ, 493, in press; astro-ph/9705081 [M32]

Young P., et al., 1978, ApJ, 221, 721

Young P., 1980, ApJ, 242, 1232 [Y 80] 